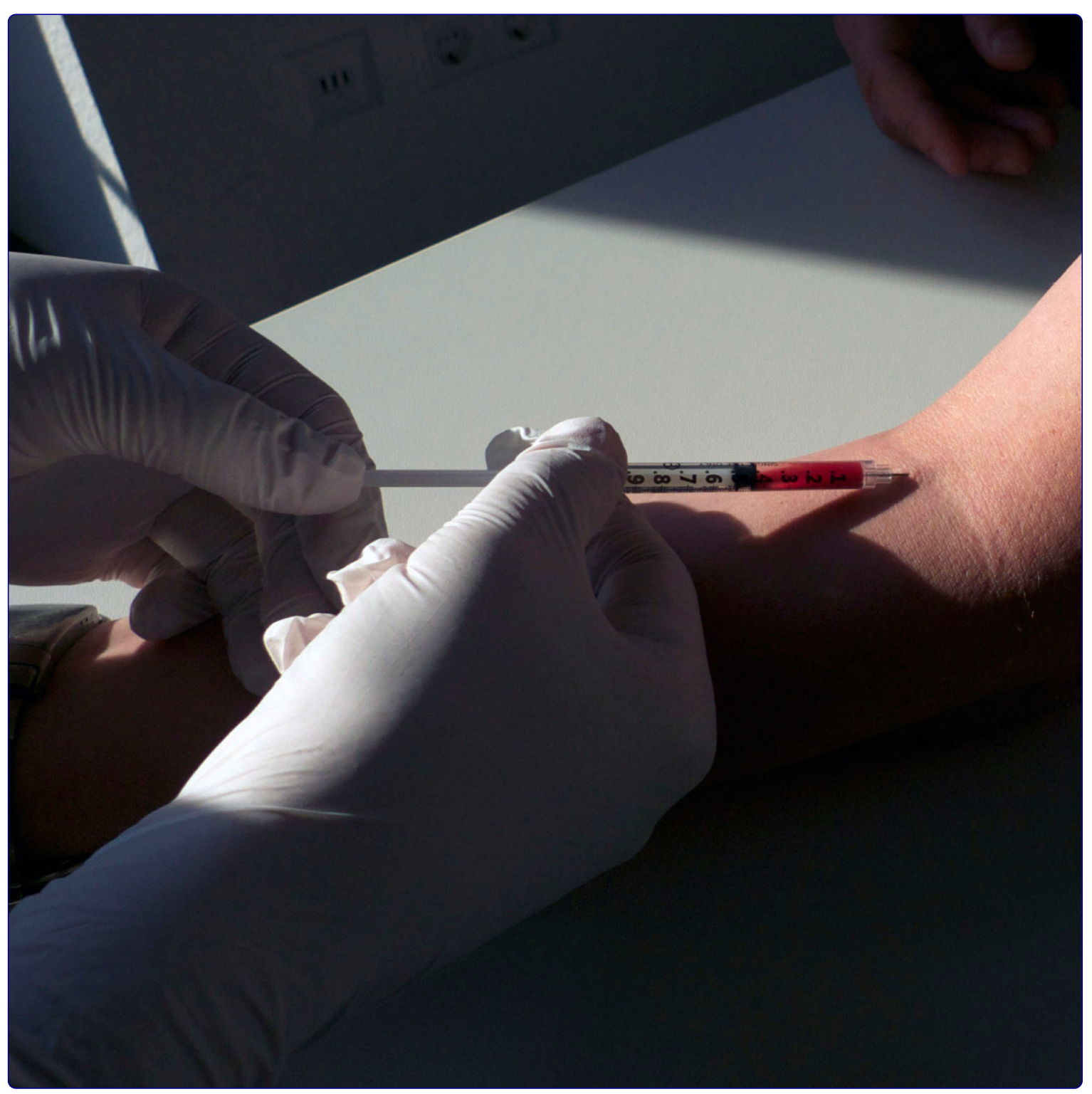

Direct venous inoculation of Plasmodium falciparum sporozoites for controlled human malaria infection: a dose-finding trial in two centres

Mordmüller et al.

C Biomed Central 


\title{
Direct venous inoculation of Plasmodium falciparum sporozoites for controlled human malaria infection: a dose-finding trial in two centres
}

Benjamin Mordmüller ${ }^{1 *}$, Christian Supan ${ }^{1}$, Kim Lee Sim², Gloria P Gómez-Pérez ${ }^{3}$, Carmen Lucelly Ospina Salazar ${ }^{1}$,

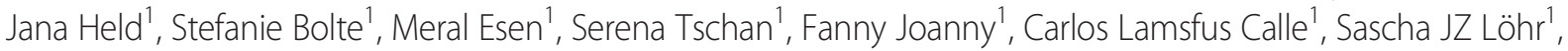
Albert Lalremruata ${ }^{1}$, Anusha Gunasekera² ${ }^{2}$ Eric R James ${ }^{2}$, Peter F Billingsley ${ }^{2}$, Adam Richman², Sumana Chakravarty ${ }^{2}$, Almudena Legarda ${ }^{3}$, Jose Muñoz ${ }^{3}$, Rosa M Antonijoan ${ }^{4,5}$, Maria Rosa Ballester ${ }^{4,5}$, Stephen L Hoffman ${ }^{2 \dagger}$, Pedro L Alonso ${ }^{3+}$ and Peter G Kremsner ${ }^{1+}$

\begin{abstract}
Background: Controlled human malaria infection (CHMI) accelerates development of anti-malarial interventions. So far, CHMl is done by exposure of volunteers to bites of five mosquitoes carrying Plasmodium falciparum sporozoites (PfSPZ), a technique available in only a few centres worldwide. Mosquito-mediated CHMl is logistically complex, exact PfSPZ dosage is impossible and live mosquito-based interventions are not suitable for further clinical development.

Methods: An open-labelled, randomized, dose-finding study in 18-45 year old, healthy, malaria-naïve volunteers was performed to assess if intravenous (IV) injection of 50 to 3,200 aseptic, purified, cryopreserved PfSPZ is safe and achieves infection kinetics comparable to published data of mosquito-mediated CHMI. An independent study site verified the fully infectious dose using direct venous inoculation of PfSPZ. Parasite kinetics were assessed by thick blood smear microscopy and quantitative real time PCR.
\end{abstract}

Results: IV inoculation with 50, 200, 800, or 3,200 PfSPZ led to parasitaemia in 1/3, 1/3, 7/9, and 9/9 volunteers, respectively. The geometric mean pre-patent period (GMPPP) was 11.2 days (range 10.5-12.5) in the 3,200 PfSPZ IV group. Subsequently, six volunteers received 3,200 PfSPZ by direct venous inoculation at an independent investigational site. All six developed parasitaemia (GMPPP: 11.4 days, range: 10.4-12.3). Inoculation of PfSPZ was safe. Infection rate and pre-patent period depended on dose, and injection of 3,200 PfSPZ led to a GMPPP similar to CHMI with five PfSPZ-infected mosquitoes. The infectious dose of PfSPZ predicted dosage of radiation-attenuated PfSPZ required for successful vaccination.

Conclusions: IV inoculation of PFSPZ is safe, well tolerated and highly reproducible. It shall further accelerate development of anti-malarial interventions through standardization and facilitation of $\mathrm{CHMI}$. Beyond this, rational dose selection for whole PfSPZ-based immunization and complex study designs are now possible.

Trial registration: ClinicalTrials.gov NCT01624961 and NCT01771848.

Keywords: Malaria, Plasmodium falciparum sporozoite, Microbial challenge, Controlled human malaria infection, Clinical trial

\footnotetext{
* Correspondence: benjamin.mordmueller@uni-tuebingen.de

${ }^{\dagger}$ Equal contributors

'Institut für Tropenmedizin, Eberhard Karls Universität Tübingen and German

Center for Infection Research, 72074 Tübingen, Germany

Full list of author information is available at the end of the article
}

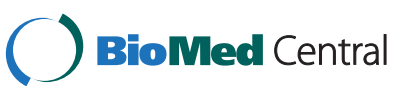

(c) 2015 Mordmüller et al.; licensee BioMed Central. This is an Open Access article distributed under the terms of the Creative Commons Attribution License (http://creativecommons.org/licenses/by/4.0), which permits unrestricted use, distribution, and reproduction in any medium, provided the original work is properly credited. The Creative Commons Public Domain Dedication waiver (http://creativecommons.org/publicdomain/zero/1.0/) applies to the data made available in this article, unless otherwise stated. 


\section{Background}

Malaria affects almost half of the world's population and it is estimated that in 2013584,000 deaths occurred [1]. The size of the problem and the ability of Plasmodium spp. to adapt rapidly to man-made interventions require expedited development of new anti-malarial tools [2]. To accelerate clinical development of antimicrobials and vaccines, human challenge models are of particular interest. Controlled human malaria infection (CHMI) with Plasmodium falciparum is among the best studied challenge models and has paved the way for many current malaria vaccine candidates [3] and some drugs for treatment and chemoprophylaxis [4]. Perhaps the main advantage of CHMI over studies under natural exposure is that it provides consistent and predictable infections, which translates to the ability to conduct simple, well-controlled trials in a small number of healthy subjects, who do not belong to a vulnerable group. This results in early, well-founded decisions on further clinical development.

$\mathrm{CHMI}$ by infected mosquitoes requires the bites of five P. falciparum sporozoite (PfSPZ)-infected mosquitoes to achieve consistent transmission, whereas one to two infected mosquitoes produce an infection rate between $50 \%[5,6]$ and $83 \%$ [7]. This led to the consensus to use five infected mosquitoes for CHMI, a number that rarely fails to induce parasitaemia in malaria-naïve volunteers $[5,8]$ and typically leads to microscopically detectable parasitaemia nine to twelve days after infection (pre-patent period). Depending on the laboratory that produces the PfSPZ-infected mosquitoes numbers required to achieve consistent infection can be lower [7,9]. Length of pre-patency varies considerably between centres [10], which is partly explained by the use of different procedures and parasite isolates. Besides the complexities of maintaining a suitable insectary, major constraints of mosquito-mediated CHMI are the restricted time window during which the mosquitoes can be used for infection, the logistic challenge of having infected mosquitoes and volunteers available at the same time, a large (and largely unknown) biological variability in the number of inoculated parasites, and the need for dissection of mosquitoes after the blood meal to prove infection and blood intake, which may require re-exposure in case the mosquitoes are negative. Direct measurement of the number of mosquito-inoculated PfSPZ in humans is not possible, and variability in pre-patent period, number of mosquitoes required for consistent infection [5,7-9] as well as vaccination success after transmission of attenuated PfSPZ $[11,12]$ suggests that PfSPZ dose is poorly controlled by counting the number of bites or mosquitoes. A potential way to overcome these constraints is injection of purified, cryopreserved, quantitated PfSPZ. In addition, such injectable PfSPZ are being developed and tested as whole-cell vaccines $[13,14]$.
Recently, manufacture of aseptic, vialed, purified, cryopreserved, infectious PfSPZ (PfSPZ Challenge) that meet regulatory standards has been achieved. In four other clinical trials, PfSPZ Challenge was administered as an intradermal (ID) or intramuscular (IM) injection and PfSPZ successfully infected human volunteers at doses between 2,500 and 25,000 PfSPZ [15-18]. However, the prepatent period was approximately two days longer than after mosquito-mediated CHMI, and with ID administration there was no dose response.

Here, the results of a CHMI study of PfSPZ Challenge administered by intravenous (IV) injection through an indwelling catheter and by direct venous inoculation (DVI) are reported. The objective of the study was to establish the minimal number of PfSPZ required to consistently infect volunteers with a pre-patent period comparable to published data on exposure to the bites of five PfSPZ-infected mosquitoes.

\section{Methods}

\section{Study design and participants}

The study was an open-labelled PfSPZ Challenge IV dose-escalation trial with an ID injection control arm (Tübingen) and a verification group at a second study site (Barcelona) to assess reproducibility of the best IV dose. Volunteers were to be healthy and malaria-naïve individuals between 18 and 45 years of age, who had no history of malaria or long-term residency in a malariaendemic area, never received an investigational malaria vaccine, were not immunosuppressed, had no acute or chronic infection or other disease, did not abuse alcohol or any other drug and belonged to a low-risk group for cardiac disease [19]. Before enrolment, written informed consent was obtained and understanding of the study and procedures was assessed with a quiz. The study received approval by the ethics committee of the University Clinic and the Medical Faculty of the University of Tübingen, and in Barcelona by the ethics committees of the Hospital Clinic and the Hospital de la Santa Creu i Sant Pau. The study followed the principles of the Declaration of Helsinki in its $6^{\text {th }}$ revision as well as International Conference on Harmonization - Good Clinical Practice (ICH-GCP) guidelines. The study is registered with ClinicalTrials.gov, numbers NCT01624961 and NCT01771848.

In Tübingen, volunteers were randomly assigned to the IV or ID arm by a computer-generated sequence provided in sealed envelopes by a third party on the day of PfSPZ Challenge injection (Day 0). The injections took place between June 12 and September 12, 2012. Safety follow-up of volunteers was done for six months. Dose-escalation of PfSPZ Challenge IV was in four-fold increases, starting with 50 and ending with a maximal dose of 3,200 PfSPZ. At each dose, three volunteers were injected and if $3 / 3$ became parasitaemic within three weeks another six were 
inoculated with the same dose. The dose was increased to the next level when less than $3 / 3$ or less than $9 / 9$ became parasitaemic. A further dose increase was planned if $9 / 9$ volunteers became parasitaemic but the geometric mean pre-patent period was greater than 12 days. ID injections were given as two separate doses of $50 \mu \mathrm{L}$ containing 1,250 PfSPZ in the deltoid region of each arm. In Tübingen, PfSPZ Challenge IV was administered as a slow injection of $0.5 \mathrm{~mL}$ via an IV catheter preceded and followed by a flush with at least $2 \mathrm{~mL}$ of physiological saline. In Barcelona, an IV catheter was inserted in the left arm to serve as emergency access. PfSPZ were administered by direct venous inoculation (DVI) of $0.5 \mathrm{~mL}$ parasite suspension into the right arm by venipuncture using a $1 \mathrm{~mL}$ syringe with $25 \mathrm{G} \times 16 \mathrm{~mm}$ needle on April 19, 2013 .

\section{PfSPZ Challenge}

PfSPZ Challenge contains aseptic, purified, cryopreserved NF54 PfSPZ, isolated from Anopheles stephensi mosquitoes, reared and infected under aseptic conditions $[14,20]$. NF54 is susceptible to all clinically used antimalarials and has been used extensively in CHMI experiments [8]. PfSPZ Challenge was kept at -195 to $-150^{\circ} \mathrm{C}$ in liquid nitrogen vapour phase. Two separate lots of PfSPZ Challenge, produced 16 months apart (March 2011 and July 2012), were used in the study. PfSPZ Challenge released for clinical use meets quality control specifications including sterility, purity and potency $[14,20]$. The quality control release and stability programme assessed potency and viability using in vitro infection of cultured human hepatocytes (HC-04) and a membrane integrity assay (Table 1), respectively as described [14,20]. Briefly, 50,000 PfSPZ were added to 40,000 HC-04 (1F9) cells and cultured for six days. Late liver stage parasites were detected by staining with a monoclonal antibody against $P$. falciparum merozoite protein 1 . Membrane integrity was tested by fluorescence microscopy of PfSPZ following incubation with SYBR green and propidium iodide. Volunteers were inoculated within 30 minutes after thawing of PfSPZ Challenge.

\section{Procedures}

All volunteers were observed for at least one hour after PfSPZ Challenge administration (Day 0) and examined on the subsequent day (Day 1) followed by daily telephone or electronic mail contacts. In Tübingen, twicedaily visits and thick blood smears were performed from Day 5 until the first thick blood smear was positive or Day 21 was reached. In Barcelona, once-daily visits were performed between Days 6 and 9, followed by twicedaily visits between Days 10 and 15. Quantitative thick blood films were prepared as described [21] at least once a day. Two or more microscopists were required to observe a minimum of two unambiguous parasites to declare
Table 1 In vitro infectivity to a hepatocyte line (HC-04) (potency) and sporozoite membrane integrity (viability) of the two lots of PfSPZ Challenge

\begin{tabular}{|c|c|c|}
\hline \multicolumn{3}{|l|}{ A) Tübingen } \\
\hline Release date & $\begin{array}{l}\text { Potency } \pm \text { standard deviation } \\
\text { (No. of parasites expressing } \\
\text { PfMSP-1/well) }\end{array}$ & $\begin{array}{l}\text { Viability } \pm \text { standard } \\
\text { deviation }\end{array}$ \\
\hline Fresh* & $32.7 \pm 1.5$ parasites & $98.2 \%$ \\
\hline Release $^{* *}$ & $29.3 \pm 3.1$ parasites & $87.4 \% \pm 5.9 \%$ \\
\hline 3 months & $27.3 \pm 0.6$ parasites & $84.6 \% \pm 1.9 \%$ \\
\hline 6 months & $26.7 \pm 1.5$ parasites & $83.6 \% \pm 5.5 \%$ \\
\hline 9 months & $26.3 \pm 2.5$ parasites & $86.3 \% \pm 6.5 \%$ \\
\hline 12 months & $27.3 \pm 0.6$ parasites & $86.2 \% \pm 1.3 \%$ \\
\hline $\begin{array}{l}\text { Post last clinical } \\
\text { dose Tübingen }\end{array}$ & $24.0 \pm 1.7$ parasites & $81.7 \% \pm 2.6 \%$ \\
\hline \multicolumn{3}{|l|}{ B) Barcelona } \\
\hline Release date & $\begin{array}{l}\text { Potency } \pm \text { standard deviation } \\
\text { (No. of parasites expressing } \\
\text { PfMSP-1/well) }\end{array}$ & $\begin{array}{l}\text { Viability } \pm \text { standard } \\
\text { deviation }\end{array}$ \\
\hline Fresh* & $28.3 \pm 1.5$ parasites & $95.5 \%$ \\
\hline Release $^{* *}$ & $25.3 \pm 1.5$ parasites & $89 \% \pm 2.2 \%$ \\
\hline 3 months & $21.7 \pm 1.5$ parasites & $85 \% \pm 3.3 \%$ \\
\hline 6 months & $25.0 \pm 5.3$ parasites & $86 \% \pm 4.8 \%$ \\
\hline $\begin{array}{l}\text { Post last clinical } \\
\text { dose Barcelona }\end{array}$ & $19.0 \pm 1.0$ parasites & $85 \% \pm 4.4 \%$ \\
\hline
\end{tabular}

*Fresh refers to the aseptic, purified PfSPZ of this lot before they were cryopreserved. Data from all other time points were generated on thawed PfSPZ Challenge.

**Release refers to the data generated within a few weeks of manufacture that were used to demonstrate that PfSPZ Challenge met quality control "release" specifications. All other data are from the formal stability programme.

a slide positive with a limit of detection below four parasites per $\mu \mathrm{L}$. On the day of first microscopically detectable parasitaemia or Day 21 (if no parasites had been detected by then) volunteers started a curative anti-malarial treatment with artemether-lumefantrine (Tübingen) or chloroquine (Barcelona). Subjects were considered cured when two consecutive thick blood smears were negative and symptoms ceased. Later follow-up visits of volunteers occurred on Days 28, 84 and 168 after inoculation in Tübingen, and on Days 35 and 90 in Barcelona. Adverse events (AE) and clinical symptoms were reviewed daily until Day 21 and on all follow-up visits thereafter.

DNA from blood was isolated before PfSPZ Challenge administration and every second day beginning on Day 5 in Tübingen or every time a blood smear was taken to perform a thick blood smear in Barcelona. Parasitaemia was estimated by quantitative polymerase chain reaction (qPCR) as described previously [22]. DNA extraction of blood samples and a dilution from ring stage parasite culture was done in the presence of an extraction control (DEC 610, Bioline) using silica spin columns (Qiagen). Amplification and detection of fluorescence was done with a RotorGene 3000 (Corbett). Limit of quantification 
of qPCR was 30 parasites per $\mathrm{mL}$. In contrast to thick blood smear, qPCR procedures were not fully validated and were considered exploratory. All qPCR runs were performed after completion of the trial.

\section{Objectives}

The primary objective of the study was to identify a PfSPZ Challenge dose that safely infects $9 / 9$ volunteers after intravenous injection and the secondary objective was to assess if increasing the PfSPZ Challenge dose results in a pre-patent period of twelve or less days. Successful infection was defined as the appearance of asexual parasites in peripheral blood, detected by thick blood film microscopy. Upon completion of the trial in Tübingen it was clear that the objectives regarding infection rate and pre-patent period had been achieved. It was, therefore, decided to verify reproducibility of the successful dose of 3,200 PfSPZ IV in an independent group $(n=6)$ at a different study site in Barcelona, and to use DVI instead of injection through an in-dwelling catheter to administer PfSPZ Challenge.

\section{Statistical analysis}

A one parameter exponential model was used to model the effect of dose on the probability of infection [23]. The effect of dose on length of pre-patent period was modelled under the assumptions that volunteers who did not develop parasitaemia until Day 21 had no risk of developing parasitaemia thereafter and that the relationship between dose and pre-patent period was linear. Parasitaemia on the day of first positive thick blood smear was used as a covariate in the model. Dose and parasitaemia were $\log _{10^{-}}$ transformed. PCR data were used to estimate parasite multiplication rates using a mixed linear model with volunteer as random variable and PfSPZ Challenge dose and time as independent variables. Safety and tolerability data were analysed by descriptive and visual methods following published guidelines and grading schemes for clinical and laboratory abnormalities [24]. Calculations were done with $\mathrm{R}$ version 2.15 .2 [25] and a two-sided $\mathrm{p}<0.05$ was considered statistically significant.

\section{Results}

\section{Dose escalation of intravenous PfSPZ Challenge}

A total of 30 volunteers with similar demographic characteristics were included in the dose-escalation phase of the trial (Table 2). Six received 2,500 PfSPZ Challenge ID and 24 IV (Figure 1). PfSPZ Challenge ID led to four successful infections with a geometric mean pre-patent period of 13.6 days (Table 3). The infection rate and prepatent period of PfSPZ Challenge ID were comparable to what was achieved with 2,500 PfSPZ ID in previous studies $[16,15]$. The PfSPZ Challenge IV dose was increased sequentially from $50(n=3)$, to $200(n=3)$, to $800(\mathrm{n}=9)$ and finally to $3,200(\mathrm{n}=9)$ PfSPZ. Injection of 50, 200 and 800 PfSPZ IV led to asexual erythrocytic stage parasitaemia in $1 / 3,1 / 3$ and $7 / 9$ volunteers, respectively. In contrast, injection of 3,200 PfSPZ led to asexual erythrocytic stage parasitaemia in 9/9 (100\%) volunteers (Table 3). Statistical modelling of probability of infection (Figure 2) with an exponential model [23] estimates an 50\% infectious dose of 326 PfSPZ (95\% confidence interval [CI]: 169-662).

A geometric mean pre-patent period not longer than twelve days was observed in the $800 \mathrm{PfSPZ}$ (11.7 days) and 3,200 PfSPZ (11.2 days) groups (Figure 3). In the IV groups, dose, corrected for parasite density, explained $73 \%$ of variability in pre-patent period and every tenfold increase in dose of PfSPZ led to a 36 (95\% CI: 23-48) hours reduction in pre-patent period. This is in contrast to published data [26] on the dose-response relationship between number of mosquitoes and pre-patent period (Figure 4).

\section{Dose verification}

Six volunteers received 3,200 PfSPZ Challenge by DVI by an independent team in Barcelona. All (6/6) became parasitaemic with a geometric mean of 11.4 days (Table 3). All volunteers at both sites were treated with an anti-malarial, either when thick blood smears became positive or on Day 21 in those who still had a negative thick blood smear.

\section{Early parasite kinetics measured by qPCR}

All blood smear results were confirmed by qPCR and none of the microscopically negative volunteers reached the positivity threshold. On average, qPCR detected parasites 65 (range 2-167) hours before microscopy. The pattern of parasite multiplication did not show pronounced synchronicity (Figure 5). The estimated parasite multiplication rate per 48 hours until the first positive thick blood smear, was 10.2 (95\% CI 5.0-21.0).

\section{Safety and tolerability of PfSPZ Challenge}

Overall, IV injection of PfSPZ Challenge through a catheter and by DVI was very well tolerated. Nevertheless, one volunteer (800 PfSPZ group) experienced mild nausea ten hours after injection, which was considered possibly related to PfSPZ Challenge administration. No other individual experienced a PfSPZ injection-related AE. A total of 28 volunteers developed parasitaemia detected by thick blood smear and all developed at least one symptom characteristic of malaria. A total of 286 adverse events (AE) occurred in 34 of the 36 volunteers. The vast majority of AEs happened around the time when parasitaemia became detectable by microscopy (Figure 6). Most AEs were mild (Grade 1; $\mathrm{n}=232$ ). Fourteen volunteers experienced 
Table 2 Demographic characteristics of the participants

\begin{tabular}{llllllll}
\hline Variable & Total & ID 2500 & IV $\mathbf{5 0}$ & IV 200 & IV 800 & IV 3200 & DVI 3200 \\
\hline $\mathrm{N}$ & 36 & 6 & 3 & 3 & 9 & 9 & 6 \\
Age in years* & $26(19 ; 43)$ & $24(21 ; 42)$ & $24(23 ; 27)$ & $27(27 ; 32)$ & $26(21 ; 43)$ & $27(24 ; 30)$ & $29(19 ; 40)$ \\
Gender, Female:Male\# & $11: 25$ & $1: 5$ & $0: 3$ & $0: 3$ & $4: 5$ & $2: 7$ & $4: 2$ \\
Height in cm* & $176(159 ; 196)$ & $178(166 ; 196)$ & $186(178 ; 190)$ & $169(167 ; 186)$ & $177(166 ; 189)$ & $176(163 ; 196)$ & $167(159 ; 172)$ \\
Weight in kg* & $73(55 ; 111)$ & $83(63 ; 92)$ & $77(70 ; 100)$ & $77(68 ; 82)$ & $74(59 ; 90)$ & $71(64 ; 111)$ & $61.1(55 ; 73)$ \\
BMI in kg/m* & $23.9(18.5 ; 29.4)$ & $24.1(22.9 ; 29.4)$ & $22.1(21.3 ; 28.9)$ & $24.3(22.3 ; 28.7)$ & $23.8(19.1 ; 26.6)$ & $24.1(18.5 ; 28.8)$ & $22.8(19.1 ; 24.8)$ \\
Hb in $\mathrm{g} / \mathrm{dl}^{*}$ & $14.7(11.8 ; 16.7)$ & $15.1(12.5 ; 15.3)$ & $15.6(15.1 ; 16.2)$ & $16.1(14.7 ; 16.7)$ & $14.2(12.0 ; 16.6)$ & $14.6(11.8 ; 15.7)$ & $13.3(12.3 ; 15.1)$ \\
Platelets/nL & $248(138 ; 396)$ & $231(172 ; 260)$ & $237(138 ; 340)$ & $263(210 ; 307)$ & $221(176 ; 261)$ & $246(193 ; 396)$ & $321(259 ; 364)$ \\
\hline
\end{tabular}

${ }^{*}$ Median (min; max), ${ }^{*} \mathrm{~N}$, ID: intradermal, IV: intravenous (dose-escalation group), DVI: direct venous inoculation (verification group).

moderate (Grade 2) and six severe (Grade 3) AEs, respectively (Table 4). No serious AE (SAE) occurred.

The most frequent $\mathrm{AE}$ was headache $(\mathrm{n}=58,6$ Grade 2). This was followed by fatigue ( $\mathrm{n}=35,5$ Grade 2$)$ and fever or fever-associated symptoms ( $\mathrm{n}=32,9$ Grade 2, 2 Grade 3). Laboratory abnormalities included liver enzyme increases $(n=7)$, lymphopenia $(n=8)$ and bilirubinuria $(\mathrm{n}=2)$. All resolved uneventfully and there were no delayed onset AEs.

\section{Discussion}

Two previous studies have shown that PfSPZ Challenge can infect malaria-naïve volunteers following ID [16] and IM [15] injection but parasite kinetics, number of PfSPZ required and infection rate are different from mosquito-mediated CHMI. The present study found that 3,200 aseptic, purified, cryopreserved PfSPZ administered by IV injection consistently infected all subjects $(15 / 15)$ with $P$. falciparum malaria and resulted in a pre- patent period of 10.4-12.5 days, which is comparable to the pre-patent period often observed in volunteers exposed to the bites of five PfSPZ (NF54)-infected mosquitoes $[13,14,27]$. It also demonstrated that the infectivity increased from $33 \%$ to $100 \%$ and the geometric mean pre-patent period was reduced from $>13$ days to 11.2 days as the dose of PfSPZ was increased from 50 to 3,200. Importantly, IV administration of PfSPZ Challenge was safe and well tolerated and the results were reproduced at another site that used a different lot of PfSPZ, a different clinical team, and a simplified method of injection (DVI).

Kinetics of mosquito- and IV PfSPZ Challenge-mediated CHMI are very comparable. Mosquitoes deposit PfSPZ in blood vessels and into the skin, where some of them get access to the vascular system [28]. Since it has been suggested that the "skin stage" of malaria has an important role in immunity [29], it would be interesting to compare immune responses to IV and mosquito-mediated $P$. falciparum malaria in direct comparison. However, it should be

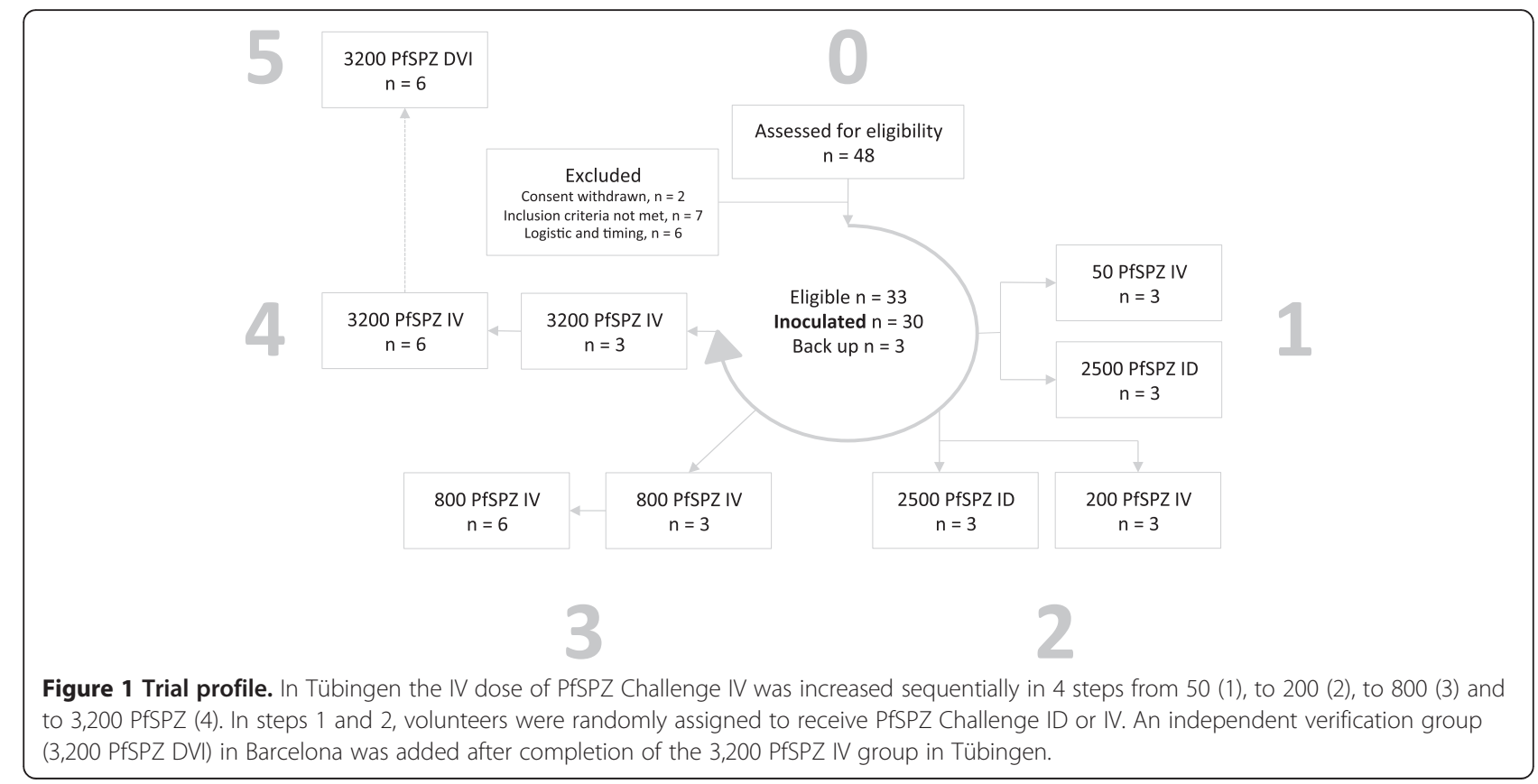


Table 3 Infection rate, pre-patent period and time to malaria

\begin{tabular}{lllll}
\hline Group & Inoculated $(\mathbf{N})$ & Parasitaemic $(\mathbf{N})$ & Prepatent period in days* (Days) & Incubation period $^{\ddagger}$ (Days) \\
\hline ID 2500 & 6 & 4 & $13.6(12.3-15.3)$ & $14.2(13.0-16.0)$ \\
IV 50 & 3 & 1 & $13.3(\mathrm{NA})$ & $7.5(\mathrm{NA})$ \\
IV 200 & 3 & 1 & $13.9(\mathrm{NA})$ & $15.0(\mathrm{NA})$ \\
IV 800 & 9 & 7 & $11.7(10.9-12.5)$ & $12.0(11.0-13.5)$ \\
IV 3200 & 9 & 9 & $11.2(10.5-12.5)$ & $9.5(7.0-12.5)$ \\
DVI 3200 & 6 & 6 & $11.4(10.4-12.3)$ & $10.6(10.0-12.0)$ \\
\hline
\end{tabular}

*Time from inoculation to first positive thick blood smear, given as geometric mean (min-max).

${ }^{\ddagger}$ Time from inoculation to first symptom judged at least possibly related to malaria, given as geometric mean (min-max).

NA: not applicable, ID: intradermal, IV: intravenous, DVI: direct venous inoculation (verification group).

noted that beginning with the seminal report on protection of mice against malaria by immunization with radiation attenuated sporozoites in 1967 [30], almost all work in animal models to develop and understand radiation attenuated sporozoite-induced immunity has used IV immunization and IV challenge. Furthermore, it has recently been shown that mosquitoes directly cannulate small vessels when feeding [31].

Notwithstanding the "non-natural" mode of administration, several important advantages emanate from the successful translation of mosquito-mediated to mosquito-free inoculation of PfSPZ for CHMI: I) improved standardization, II) exact dosing and dose-estimation for immunization studies and III) facilitated global access to CHMI in times where testing capacities shall be expanded, particularly to endemic regions.

Standardization of CHMI is central for comparison of studies between and within clinical sites and populations. In the current study, this topic was addressed by introduction of an independent verification group, located about $1000 \mathrm{~km}$ away, assessed by a different clinical and parasitological team that used DVI instead of IV injection via catheter. In addition, two different PfSPZ Challenge lots, produced 16 months apart were used. Despite these obvious differences, results were highly comparable, showing that the technique is not centre-dependent

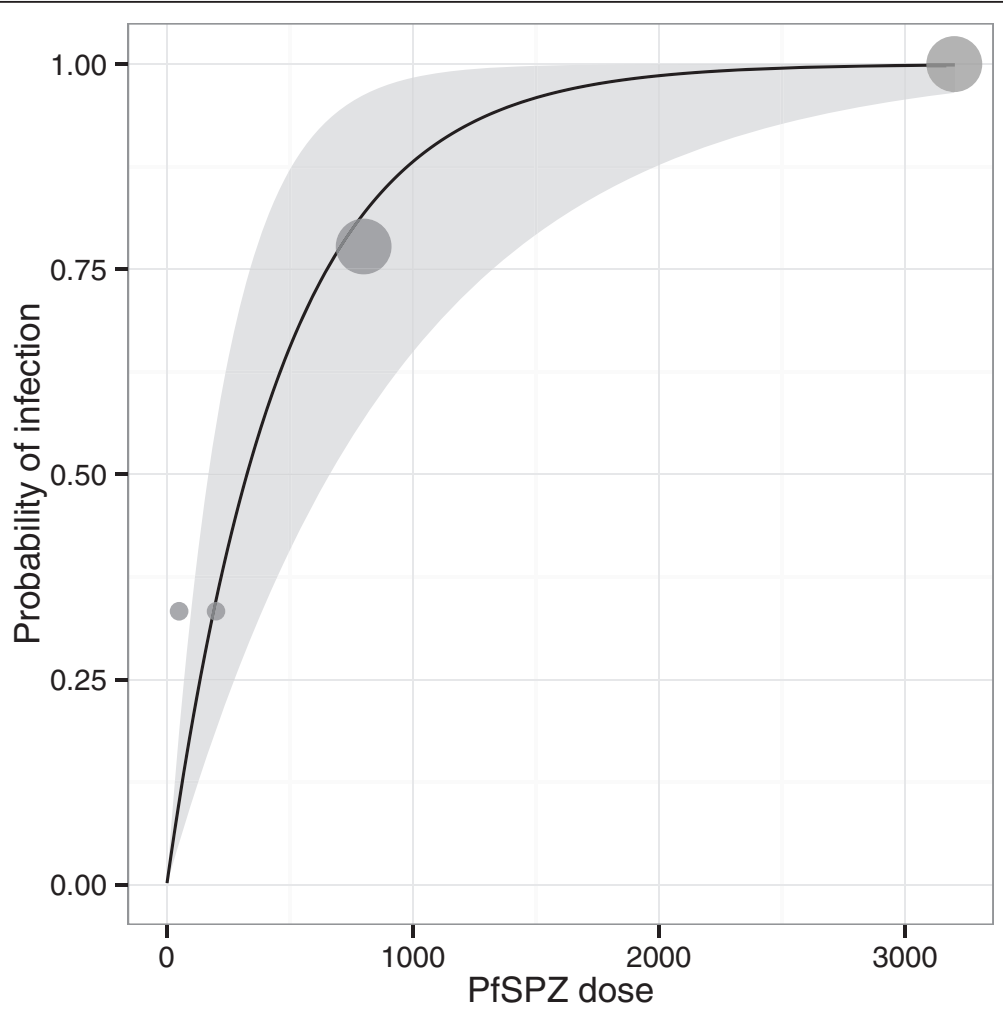

Figure 2 Effect of IV PfSPZ Challenge dose on probability of infection. Observed values are given as grey dots, with size representing weight. Model estimates are represented as the black line and the $95 \%$ confidence interval as grey ribbon. 


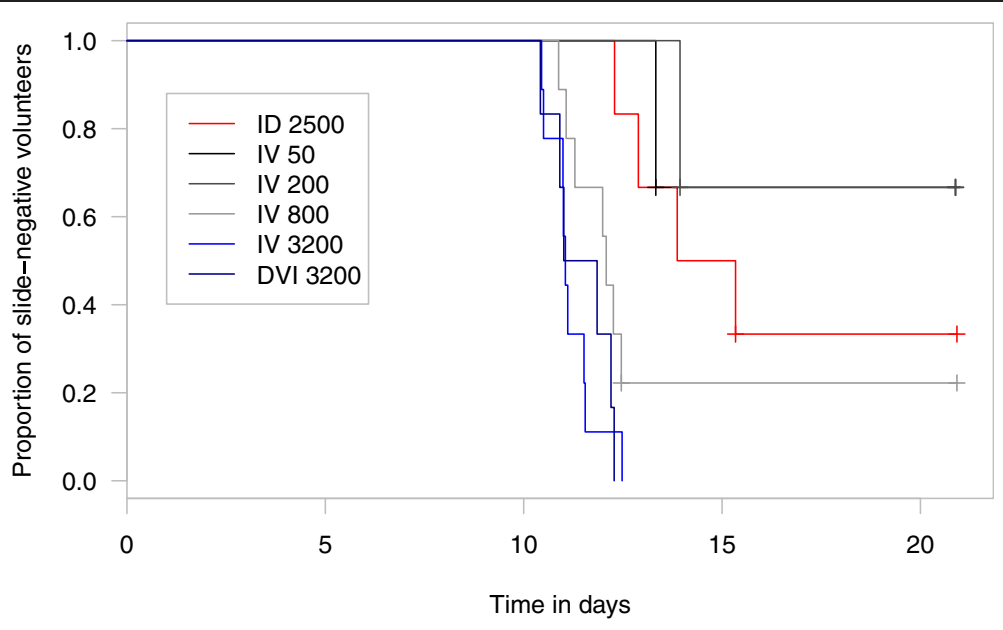

Figure 3 Kaplan-Meier plot of time to infection.

and amenable to multicentre studies, which would be hardly possible using mosquito-mediated CHMI [10].

Conversion of number of mosquitoes into PfSPZ dose cannot be exact. Nevertheless, data of this study helps to improve dose-estimation for immunization trials. Immunization by IV injection of irradiated PfSPZ (PfSPZ
Vaccine) requires a cumulative dose of 675,000 PfSPZ to protect 6/6 volunteers, whereas 540,000 PfSPZ provide only partial protection (6/9) [13]. When using infected and irradiated mosquitoes 1,000 bites are needed for protection [12,32]. Hence one mosquito transmits more than 540 and up to 675 PfSPZ Vaccine equivalents. This

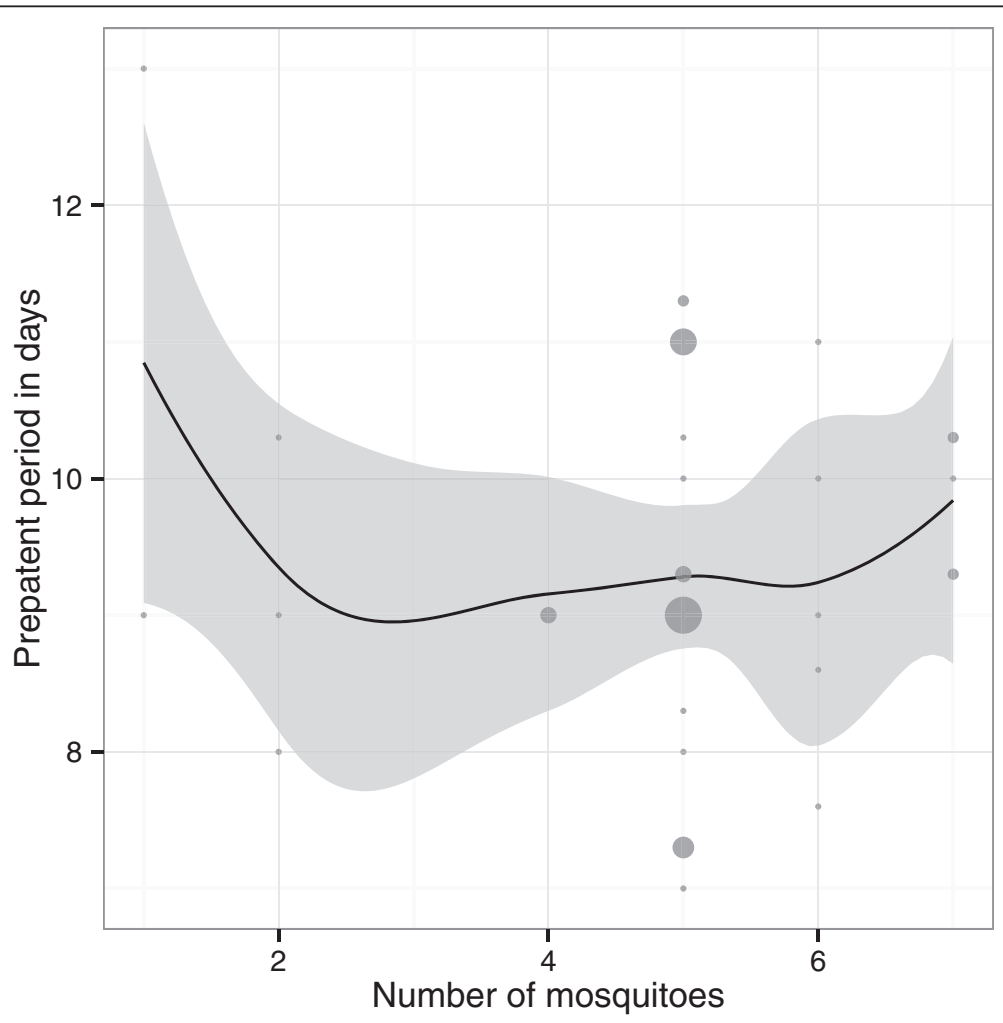

Figure 4 Effect of number of mosquito bites on pre-patent period. Published data are from 44 volunteers bitten by 1-7 infected mosquitoes at one centre (Department of Medical Microbiology, Radboud University Nijmegen Medical Centre, Nijmegen, The Netherlands) [26]. Observed values are given as grey dots with size representing weight. A Loess smooth is displayed with the estimate (black line) and $95 \%$ confidence interval (grey ribbon). 


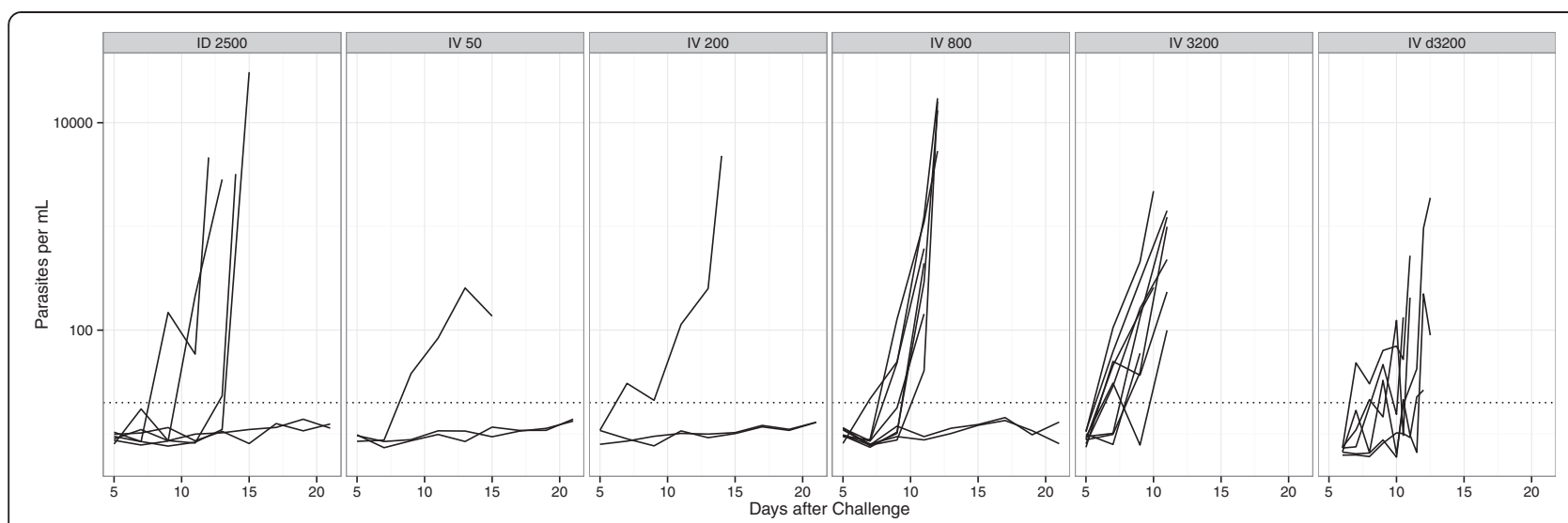

Figure 5 Parasite quantification by qPCR. Blood was assessed every other day beginning on Day 5 and on the day of microscopically detected parasitaemia if no sampling was scheduled for that day in Tübingen. In Barcelona blood was sampled every day from Days 6 to 9 and twice daily from Day 9 onward. The dotted line indicates the limit of quantification of qPCR (30 parasites per $\mathrm{mL}$ ).

matches exactly the estimate of the present CHMI study: five mosquitoes with $640 \mathrm{PfSPZ}$ Challenge equivalents per mosquito. The task of rational dosing becomes even more complicated when the biologically more relevant variable "successful passage through the liver" is considered: Assuming a 10.2-fold multiplication of asexual parasites and 10,000 successful erythrocyte invasions per infected hepatocytes in all volunteers of this study, 3,200 PfSPZ successfully infected 13 (95\% CI: 8-21) hepatocytes. This extrapolation is likely inaccurate but shall stimulate further research and technological progress, since the infected hepatocyte is a crucial immunogen in PfSPZ-based immunization strategies and hence shall be directly monitored to ensure high-level protection. Results of the first

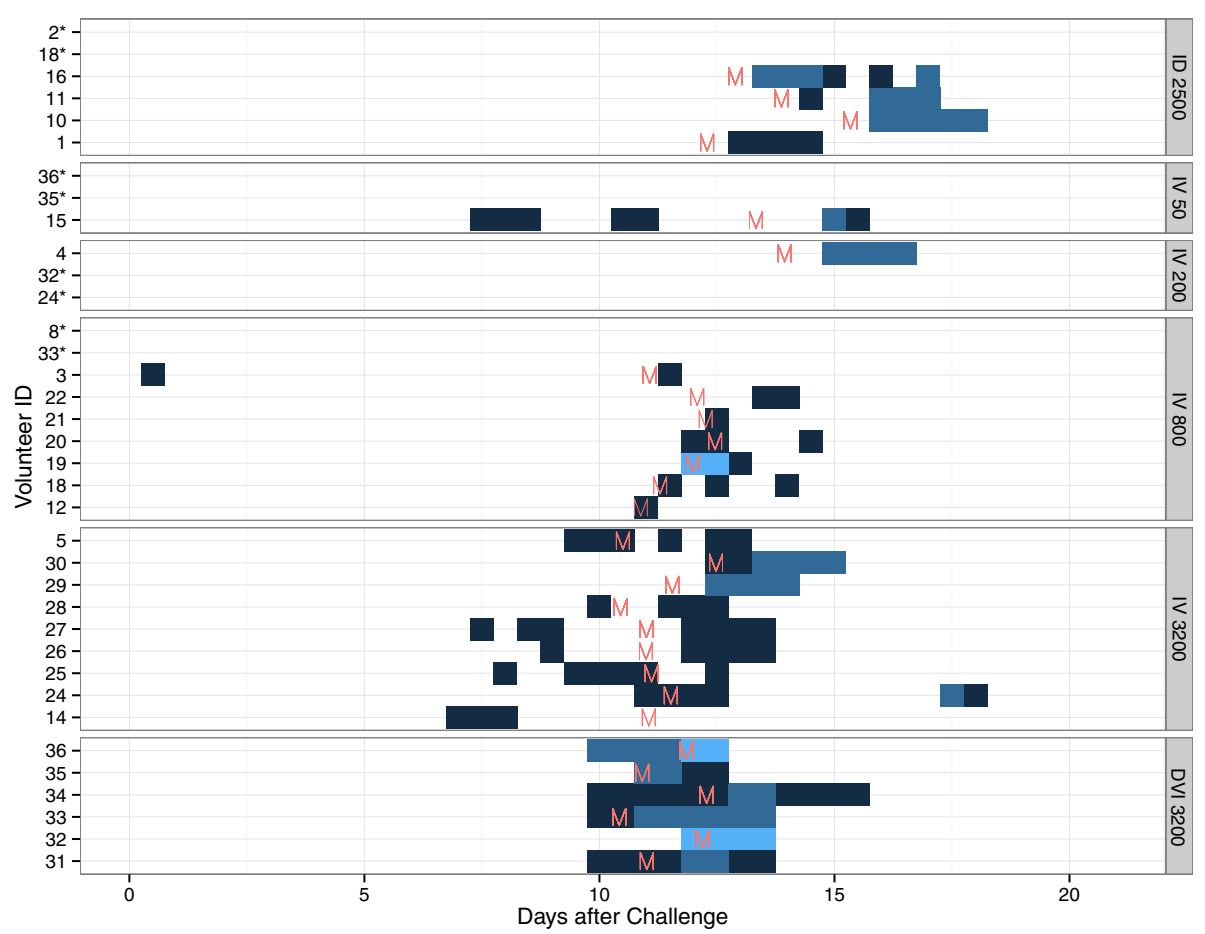

Figure 6 Adverse event pattern. Adverse event (AE) episodes are given over time from Day 0 through Day 21. Shades of blue represent AE grading from Grade 1 (dark), to Grade 2 (blue), to Grade 3 (light blue). Volunteer IDs with a star indicate those volunteers who did not develop parasitaemia. The time of parasite detection by microscopy is given as the letter ' $\mathrm{M}$ ' in red. 
Table 4 Grade 2 and Grade 3 adverse events

\begin{tabular}{|c|c|c|}
\hline Group & Grade 2* & Grade 3* \\
\hline \multirow[t]{5}{*}{ ID $2500(n=6)$} & Fatigue (2) & None \\
\hline & Fever (1) & \\
\hline & Headache (2) & \\
\hline & Tachycardia (2) & \\
\hline & High ALT (1) & \\
\hline IV $50(n=3)$ & Fever (1) & None \\
\hline \multirow[t]{3}{*}{ IV $200(n=3)$} & Fever (1) & None \\
\hline & Headache (1) & \\
\hline & Tachycardia (1) & \\
\hline IV $800(n=9)$ & None & Lymphopenia (1) \\
\hline \multirow[t]{2}{*}{ IV $3200(n=9)$} & Fatigue (2) & None \\
\hline & Fever (2) & \\
\hline \multirow[t]{19}{*}{ DVI $3200(n=6)$} & Anxiety (2) & High CRP (1) \\
\hline & Contusion (1) & \\
\hline & Common cold (1) & \\
\hline & Fatigue (1) & \\
\hline & Fever (4) & Fever (2) \\
\hline & Headache (3) & \\
\hline & High ALT (2) & \\
\hline & High AST (1) & \\
\hline & High CRP (1) & \\
\hline & Insomnia (1) & \\
\hline & Low potassium (1) & Lymphopenia (3) \\
\hline & Malaise (1) & \\
\hline & Menstrual cramps (1) & \\
\hline & Myalgia (4) & \\
\hline & Nausea (2) & \\
\hline & Neutropenia (1) & \\
\hline & Stye (1) & \\
\hline & Vomiting (2) & \\
\hline & Weakness (1) & \\
\hline Total N & 47 & 7 \\
\hline
\end{tabular}

Note that volunteers in the verification group underwent a more extensive blood-sampling scheme for laboratory parameters. Laboratory parameters were graded using an adapted U.S. FDA toxicity scale.

*Symptom (N), ID: intradermal, IV: intravenous, DVI: direct venous inoculation (verification group).

trial, where this a priori knowledge has been used to dose PfSPZ Challenge for the chemoprophylaxis with sporozoites approach [33,34], are expected in early 2015 [35].

So far, CHMI with mosquitoes is restricted to a small number of centres globally. Development of a standardized protocol for CHMI with PfSPZ Challenge enables every malaria-experienced centre to perform CHMI studies to assess anti-malarial drugs and vaccines, diagnostics, and innate and acquired resistance to malaria. Due to the high reproducibility and temporal independence, previously unfeasible study designs can be realized; from complex early phase (e.g. sequential and adaptive) to large multicentre trials in populations with very low natural exposure (e.g. travellers and populations in pre-eradication settings) and studies in endemic countries. In fact, the first CHMI trial using PfSPZ Challenge IV is underway in Gabon and several parallel trials are in progress to assess PfSPZ Vaccine IV in the United States, Europe and Africa.

\section{Conclusions}

Exposure to five infected mosquitoes is a standard technique for controlled human malaria infection (CHMI) in humans. Successful translation of mosquito-administered to injectable Plasmodium falciparum sporozoite (PfSPZ) mediated CHMI is an important step in standardization and harmonization of CHMI. It allows complex and larger multicentre trials and increases the number of groups with access to CHMI in times of active development of novel preventive and therapeutic interventions. CHMI using intravenous inoculation of PfSPZ Challenge is safe, well tolerated, highly reproducible and shall boost the understanding of malaria and the development of novel antimalarial interventions.

\section{Competing interests}

BM, CS, CLOS, JH, SB, ME, ST, FJ, CLC, SJZL, ALa, PGK, GPGP, ALe, JM, RMA, $M R B$ and PLA have no competing financial interests. BKLS, AG, ERJ, PFB, AR and SC are employees of Sanaria Inc. SLH is Chief Executive and Scientific Officer of Sanaria Inc.

\section{Authors' contributions}

BM designed the Tübingen trial, prepared the figures and drafted the manuscript. ALe, PLA and SLH designed the Barcelona trial. JH, ME, ST, FJ, $C L C, B K L S, E R J, P F B, A R$ and SC were involved in laboratory aspects of the study. BM, CS, CLOS, SB, SJZL, GPGP, ALa, JM, RMA, MRB and PLA collected the data. BM, PGK, PLA and SLH analysed the data. All authors interpreted the data, wrote the manuscript and have seen and approved the final version.

\section{Acknowledgements}

We thank all volunteers and staff of the Institute of Tropical Medicine in Tübingen, the Barcelona Centre for International Health Research and Sanaria Inc. in Rockville. Special thanks to Yonas Abebe, Anthony Ajua, Marta Aldea, John J. Aponte, Carlota Dobaño, Abraham Eappen, Marta Fernández, Tobias Gabele, Mireia Gonzáles, Alexander Hoffman, Sankarganesh Jeyaraj, Annette Knoblich, Minglin Li, Tao Li, Anita Manoj, Mireia Navarro, Carlos Arlindo Nhamuave, Josefina Pardos, Anna Pascual, Adam Ruben, Richard Stafford, Nadin Theurer, Robert C. Thompson, Soundarapandian Velmurugan, Ilka Westermann and Cecilia Justino Zita for support and help. We appreciate the advice of David Diemert, Barney Graham, Wolfram G. Metzger and Antoni Trilla, members of the Safety Monitoring Committee. Gloria P. Gómez-Pérez holds a European Commission - Erasmus Mundus Joint Doctorate Programme scholarship. The study was supported by the Federal Ministry of Education and Research in the framework of the German Center for Infection Research (DZIF) and by the Instituto de Salud Carlos III (ISCIII) and Fundación Ramón Areces, in Spain. Sanaria's research reported in this publication was supported by the National Institute of Allergy and Infectious Diseases of the National Institutes of Health under SBIR award number R01GM9987654.

\section{Author details}

'Institut für Tropenmedizin, Eberhard Karls Universität Tübingen and German Center for Infection Research, 72074 Tübingen, Germany. ${ }^{2}$ Sanaria Inc, Rockville, MD 20850, USA. ${ }^{3}$ Barcelona Center for International Health Research (CRESIB), Hospital Clínic, University of Barcelona, E-08036 Barcelona, 
Spain. ${ }^{4}$ Drug Research Center (CIM), Institute of Biomedical Research (IIB), Research Institute of Santa Creu and Sant Pau Hospital, Barcelona, Spain. ${ }^{5}$ Department of Pharmacology and Therapeutics, Autonomous University of Barcelona (UAB), Santa Creu and Sant Pau Hospital, E-08026 Barcelona, Spain.

Received: 15 November 2014 Accepted: 1 March 2015 Published online: 18 March 2015

\section{References}

1. WHO. World Malaria Report 2014. Geneva: World Health Organization; 2014.

2. Mordmüller B, Kremsner PG. Malarial parasites vs. antimalarials: never-ending rumble in the jungle. Curr Mol Med. 2006;6:247-51.

3. Sauerwein RW, Roestenberg M, Moorthy VS. Experimental human challenge infections can accelerate clinical malaria vaccine development. Nat Rev Immunol. 2011:11:57-64.

4. Fairley $\mathrm{H}$. Chemotherapeutic suppression and prophylaxis in malaria. Trans $\mathrm{R}$ Soc Trop Med Hyg. 1945;38:311-55.

5. Verhage DF, Telgt DSC, Bousema JT, Hermsen CC, van Gemert GJA, van der Meer JWM, et al. Clinical outcome of experimental human malaria induced by Plasmodium falciparum-infected mosquitoes. Neth J Med. 2005;63:52-8.

6. Rickman LS, Jones TR, Long GW, Paparello S, Schneider I, Paul CF, et al. Plasmodium falciparum-infected Anopheles stephensi inconsistently transmit malaria to humans. Am J Trop Med Hyg. 1990;43:441-5.

7. Lyke KE, Laurens M, Adams M, Billingsley PF, Richman A, Loyevsky M, et al. Plasmodium falciparum malaria challenge by the bite of aseptic Anopheles stephensi mosquitoes: results of a randomized infectivity trial. PLoS One. 2010;5:e13490

8. Epstein JE, Rao S, Williams F, Freilich D, Luke T, Sedegah M, et al. Safety and clinical outcome of experimental challenge of human volunteers with Plasmodium falciparum-infected mosquitoes: an update. J Infect Dis. 2007;196:145-54

9. Laurens MB, Billingsley P, Richman A, Eappen AG, Adams M, Li T, et al. Successful human infection with $P$. falciparum using three aseptic Anopheles stephensi mosquitoes: a new model for controlled human malaria infection. PLoS One. 2013;8:e68969.

10. Roestenberg M, O'Hara GA, Duncan CJA, Epstein JE, Edwards NJ, Scholzen A, et al. Comparison of clinical and parasitological data from controlled human malaria infection trials. PLoS One. 2012;7:e38434

11. Hoffman SL, Goh LML, Luke TC, Schneider I, Le TP, Doolan DL, et al. Protection of humans against malaria by immunization with radiationattenuated Plasmodium falciparum sporozoites. J Infect Dis. 2002;185:1155-64.

12. Clyde DF, Most H, McCarthy VC, Vanderberg JP. Immunization of man against sporozite-induced falciparum malaria. Am J Med Sci. 1973;266:169-77.

13. Seder RA, Chang L-J, Enama ME, Zephir KL, Sarwar UN, Gordon IJ, et al. Protection against malaria by intravenous immunization with a nonreplicating sporozoite vaccine. Science. 2013;341:1359-65.

14. Epstein JE, Tewari K, Lyke KE, Sim BKL, Billingsley PF, Laurens MB, et al. Live attenuated malaria vaccine designed to protect through hepatic $C D 8^{+} \mathrm{T}$ cell immunity. Science. 2011;334:475-80.

15. Sheehy SH, Spencer AJ, Douglas AD, Sim BKL, Longley RJ, Edwards NJ, et al. Optimising controlled human malaria infection studies using cryopreserved P. falciparum parasites administered by needle and syringe. PLoS One. 2013;8:e65960

16. Roestenberg M, Bijker EM, Sim BKL, Billingsley PF, James ER, Bastiaens GJH, et al. Controlled human malaria infections by intradermal injection of cryopreserved Plasmodium falciparum sporozoites. Am J Trop Med Hyg. 2013;88:5-13.

17. Shekalaghe S, Rutaihwa M, Billingsley PF, Chemba M, Daubenberger CA, James ER, et al. Controlled human malaria infection of Tanzanians by intradermal injection of aseptic, purified, cryopreserved Plasmodium falciparum sporozoites. Am J Trop Med Hyg. 2014;91:471-80.

18. Hodgson SH, Juma E, Salim A, Magiri C, Kimani D, Njenga D, et al. Evaluating controlled human malaria infection in Kenyan adults with varying degrees of prior exposure to Plasmodium falciparum using sporozoites administered by intramuscular injection. Front Microbiol. 2014;5:686.

19. Gaziano TA, Young CR, Fitzmaurice G, Atwood S, Gaziano JM. Laboratorybased versus non-laboratory-based method for assessment of cardiovascular disease risk: the NHANES I Follow-up Study cohort. Lancet. 2008;371:923-31.

20. Hoffman SL, Billingsley PF, James E, Richman A, Loyevsky M, Li T, et al. Development of a metabolically active, non-replicating sporozoite vaccine to prevent Plasmodium falciparum malaria. Hum Vaccin. 2010;6:97-106.
21. Planche T, Krishna S, Kombila M, Engel K, Faucher JF, Ngou-Milama E, et al. Comparison of methods for the rapid laboratory assessment of children with malaria. Am J Trop Med Hyg. 2001;65:599-602.

22. Hermsen CC, Telgt DS, Linders EH, van de Locht LA, Eling WM, Mensink EJ, et al. Detection of Plasmodium falciparum malaria parasites in vivo by real-time quantitative PCR. Mol Biochem Parasitol. 2001:118:247-51.

23. Haas CN. Estimation of risk due to low doses of microorganisms: a comparison of alternative methodologies. Am J Epidemiol. 1983;118:573-82.

24. Bonhoeffer J, Bentsi-Enchill A, Chen RT, Fisher MC, Gold MS, Hartman K, et al. Guidelines for collection, analysis and presentation of vaccine safety data in surveillance systems. Vaccine. 2009;27:2289-97.

25. R Core Team. R: A Language and Environment for Statistical Computing. Vienna, Austria; 2014. [http://www.R-project.org/]

26. Turner L, Wang CW, Lavstsen T, Mwakalinga SB, Sauerwein RW, Hermsen CC, et al. Antibodies against PfEMP1, RIFIN, MSP3 and GLURP are acquired during controlled Plasmodium falciparum malaria infections in naïve volunteers. PLoS One. 2011;6:e29025.

27. Richie TL, Charoenvit Y, Wang R, Epstein JE, Hedstrom RC, Kumar S, et al. Clinical trial in healthy malaria-naïve adults to evaluate the safety, tolerability, immunogenicity and efficacy of MuStDO5, a five-gene, sporozoite/hepatic stage Plasmodium falciparum DNA vaccine combined with escalating dose human GM-CSF DNA. Hum Vaccines Immunother. 2012;8:1564-84.

28. Vanderberg JP, Frevert U. Intravital microscopy demonstrating antibodymediated immobilisation of Plasmodium berghei sporozoites injected into skin by mosquitoes. Int J Parasitol. 2004;34:991-6.

29. Sinnis $P$, Zavala F. The skin: where malaria infection and the host immune response begin. Semin Immunopathol. 2012;34:787-92.

30. Nussenzweig RS, Vanderberg J, Most H, Orton C. Protective immunity produced by the injection of $x$-irradiated sporozoites of Plasmodium berghei. Nature. 1967;216:160-2.

31. Choumet V, Attout T, Chartier L, Khun H, Sautereau J, Robbe-Vincent A, et al. Visualizing non infectious and infectious Anopheles gambiae blood feedings in naive and saliva-immunized mice. PLoS One. 2012;7:e50464.

32. Rieckmann KH, Carson PE, Beaudoin RL, Cassells JS, Sell KW. Sporozoite induced immunity in man against an Ethiopian strain of Plasmodium falciparum. Trans R Soc Trop Med Hyg. 1974;68:258-9.

33. Roestenberg M, McCall M, Hopman J, Wiersma J, Luty AJF, van Gemert GJ, et al. Protection against a malaria challenge by sporozoite inoculation. N Engl J Med. 2009;361:468-77.

34. Bijker EM, Bastiaens GJH, Teirlinck AC, van Gemert G-J, Graumans W, van de Vegte-Bolmer $\mathrm{M}$, et al. Protection against malaria after immunization by chloroquine prophylaxis and sporozoites is mediated by preerythrocytic immunity. Proc Natl Acad Sci U S A. 2013;110:7862-7.

35. Safety and protective efficacy of intravenous immunization with cryopreserved Plasmodium falciparum sporozoites under chemoprophylaxis [http:// clinicaltrials.gov/ct2/show/NCT02115516]

\section{Submit your next manuscript to BioMed Central and take full advantage of:}

- Convenient online submission

- Thorough peer review

- No space constraints or color figure charges

- Immediate publication on acceptance

- Inclusion in PubMed, CAS, Scopus and Google Scholar

- Research which is freely available for redistribution 